\title{
Enhancing One-dimensional Charge Transport through Cofacial $\pi$-Electronic Delocalization: Conductivity Improvement for Organic Nanobelts
}

\author{
Yanke Che, ${ }^{1}$ Aniket Datar, ${ }^{1}$ Xiaomei Yang, ${ }^{1}$ Tammene Naddo, ${ }^{1}$ Jincai Zhao, ${ }^{*}, 2$ and Ling Zang ${ }^{*},{ }^{1}$ \\ ${ }^{1}$ Department of Chemistry and Biochemistry, Southern Illinois University, Carbondale, IL \\ 62901; ${ }^{2}$ Key Laboratory of Photochemistry, Institute of Chemistry, Chinese Academy of \\ Sciences, Beijing 100080, China.
}

\section{Supporting Information}

\section{Preparation of molecules and nanobelts}

Two PTCDI molecules (below) were synthesized following the standard condensation method developed by Langhals ${ }^{1}$ and in our lab., ${ }^{2,3}$ PE-PTCDI was employed in this study as the target molecule to afford highly organized cofacial $\pi$-stacking, while ND-PTCDI was used as a comparison that prevents formation of cofacial stacking due to the strong side-chain interference. $^{3}$

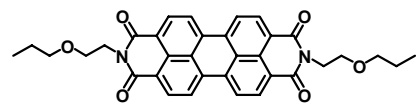

N,N’-di(propoxyethyl)-perylene-3,4,9,10-tetracarboxylic diimide (PE-PTCDI)

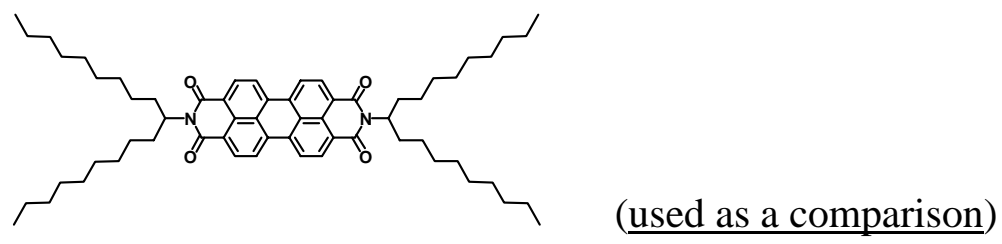

N,N’-di(nonyldecyl)-perylene-3,4,9,10-tetracarboxylic diimide (ND-PTCDI)

Nanobelts of PE-PTCDI (with large length-to-width aspect ratio) were fabricated using a slow self-assembling method, so called 'phase transfer', recently developed in our lab. ${ }^{3}$ Typically, a larger amount (10:1 vol) of methanol (a poor solvent) was transferred atop a concentrated chloroform solution of PE-PTCDI $(1 \mathrm{mM})$ in a test tube. Within minutes, red crystals formed at the interface between methanol and chloroform, followed by slow diffusion into the upper phase of methanol. The nanobelts thus formed can be transferred and cast onto glass surface by pipetting.

In contrast, ND-PTCDI is soluble in almost all the organic solvents due to the branched structure of the side-chain. To facilitate the aggregation of molecules, one has to use a mixed solvent composed of an organic solvent and water (in which the molecule is not soluble at all). In this study, the self-assembly of ND-PTCDI was performed in a binary solvent of water/methanol (35/65). Particulate aggregates in approximately spherical shape, with average 
size of $\sim 200 \mathrm{~nm}$, were obtained (Figure S3, vide infra). The spherical morphology of molecular aggregate is consistent with the distorted $\pi-\pi$ stacking, which prevents the molecules from assembling along one dimension.

\section{UV-vis absorption and fluorescence spectrometry}

UV-vis absorption and fluorescence spectra were measured on a PerkinElmer Lambda 25 spectrophotometer and LS 55 fluorometer, respectively.

Free anionic radical of PTCDI was produced by adding excess of hydrazine $(0.5 \mathrm{M})$ into a diluted solution of PTCDI $(20 \mu \mathrm{M})$ in DMF. In a deoxygenated solution (e.g., saturated with argon), the produced radicals can be accumulated till complete conversion of the neutral PTCDI molecules; within about $30 \mathrm{~min}$, the color of the solution became from bright orange to dark green, indicative of formation of anionic radicals of PTCDI (see the inset of Figure 1). UV-vis absorption measurements showed the new absorption bands emerged at longer wavelengths (600 $-1000 \mathrm{~nm}$ ) accompanying the conversion of neutral molecules into anionic radicals (see Figure 1). Consistently, the fluorescence of neutral molecules (with quantum yield $~ 100 \%$ ) decreased depending on the concentration of hydrazine added (Figure S1).

Note: since the two nitrogen positions in PTCDI molecule are node in the $\pi$-orbital wavefunction, changing the side-chains at the nitrogen position does not alter the electronic structure of PTCDI, and thus the absorption spectra at both neutral and radical states. Indeed, the same absorption and fluorescence spectra and fluorescence quenching efficiency were observed for the two PTCDI molecules in solutions. However, the stronger side-chain interference associated with ND-PTCDI distorts the molecular stacking from cofacial configuration as evidenced before, ${ }^{3}$ thus leading to different intermolecular electronic interaction. Different stacking configuration leads to different efficiency in $\pi$-electronic delocalization, as observed by the ESR and conductivity measurements in this study (vide infra).

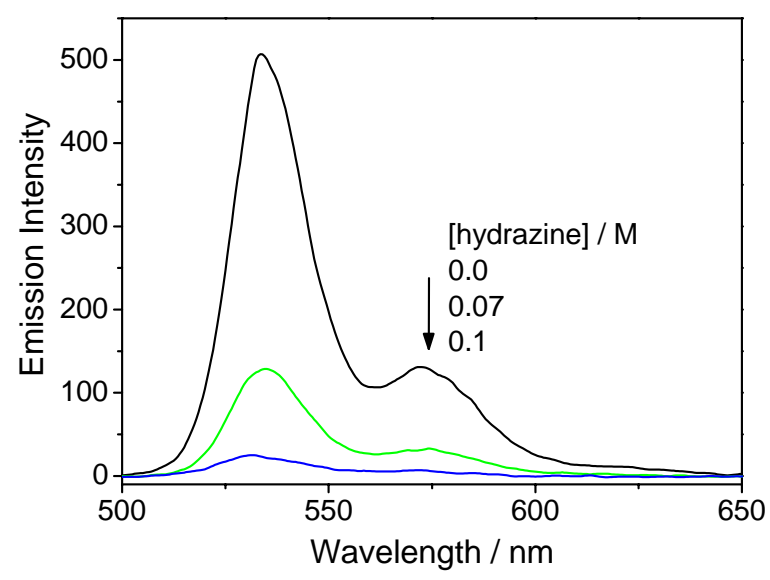

Figure S1. Fluorescence quenching of PE-PTCDI $(1.0 \mu \mathrm{M})$ by hydrazine in DMF showing efficient charge transfer between PTCDI and hydrazine.

\section{ESR measurements}

Electron spin resonance (ESR) spectra were recorded with a Bruker ESP500E spectrometer under the settings of center field, $3480.00 \mathrm{G}$, microwave frequency, $9.79 \mathrm{GHz}$, power, $5.05 \mathrm{~mW}$. 
ESR spectra of PE-PTCDI free radicals were measured in DMF solutions $(20 \mu \mathrm{M})$ in the presence of $0.5 \mathrm{M}$ hydrazine in a sealed capillary. Hyperfine ESR spectrum was obtained for the isotropic radical solution (Figure S2). The g-tensor, 2.0033, the hyperfine coupling constants, $4 \mathrm{H}$ with $\mathrm{A}=1.8 \mathrm{G}, 2 \mathrm{~N}$ with $\mathrm{A}=0.59 \mathrm{G}, 4 \mathrm{H}$ with $\mathrm{A}=0.59 \mathrm{G}$ and a line width of $0.21 \mathrm{G}$, are very close to the values reported for other PTCDI radicals. ${ }^{4}$ Same hyperfine spectra were also obtained in other solvents such as DMSO.

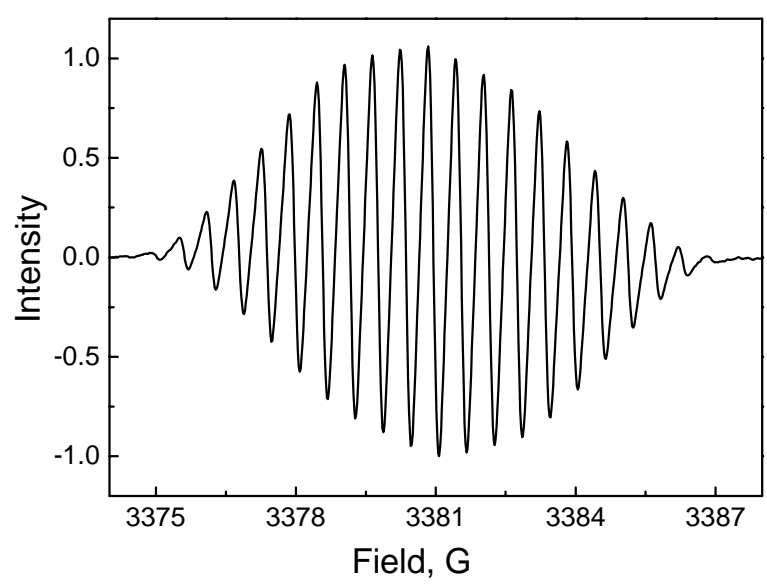

Figure S2. Hyperfine ESR spectrum of PE-PTCDI anionic radical in a DMF solution: $20 \mu \mathrm{M}$ of PE-PTCDI in the presence of excessive hydrazine.

The ESR spectra of PE-PTCDI nanobelts were measured in methanol, in which the nanobelts were suspended in the presence of excessive hydrazine $(\sim 0.5 \mathrm{M})$. The reduced PTCDI (anionic radical) thus produced is in aggregate state packed with large number of neutral molecules. As detailed in the Communication context, due to the effective cofacial stacking between PTCDI planes, the doped electron is no longer localized at one PTCDI, but delocalized along the molecular stacking. Such a 1D $\pi$-delocalization results in dramatically different ESR spectral structure for the reduced PTCDI (compared to that of free radicals) as depicted in Figure 2.

The ESR spectra of ND-PTCDI nanoparticles were measured in solid state rather than in suspension, since the particles stick strongly to the glass surface in the methanol/water binary solvent, in which the nanoparticles were prepared via precipitation. Briefly, certain amount of nanoparticles were adsorbed onto cotton from the methanol/water solution (upon sonication). The particle-contained cotton was then put into a sealed capillary, in which about $10 \mu \mathrm{L}$ of hydrazine was present. Within minutes, the cotton and the nanoparticles will be saturated with hydrazine vapor, and the reduced PTCDI was detected by ESR spectrometry.

Interestingly, the obtained ESR spectrum (Figure S3, g-tensor 2.0034) was quite similar to the spectrum measured from free radical solutions (as shown Figure 2, g-tensor 2.0033), but dramatically different from that measured with nanobelts (all shown in Figure 2). This observation is consistent with the distorted molecular stacking of ND-PTCDI, for which the strong side-chain hindrance prevents cofacial molecular stacking and thus diminishes the intermolecular electronic interaction (here the $\pi$-electronic delocalization). The weak electronic delocalization facilitates local stabilization of the doped electron at one molecule, leading to electron spin property similar to the free radicals dispersed in solutions. 
Moreover, the solid state ESR measurement was also performed for the PE-PTCDI nanobelts, and the ESR spectrum thus obtained was exactly the same as that obtained from the methanol suspension (as shown in Figure 2). This observation provides additional evidence that the electron spin property of the doped electron within the nanobelt is dramatically different from that within the nanoparticle, mainly because of the different configuration and strength of $\pi$ electronic interaction between the component PTCDI molecules.
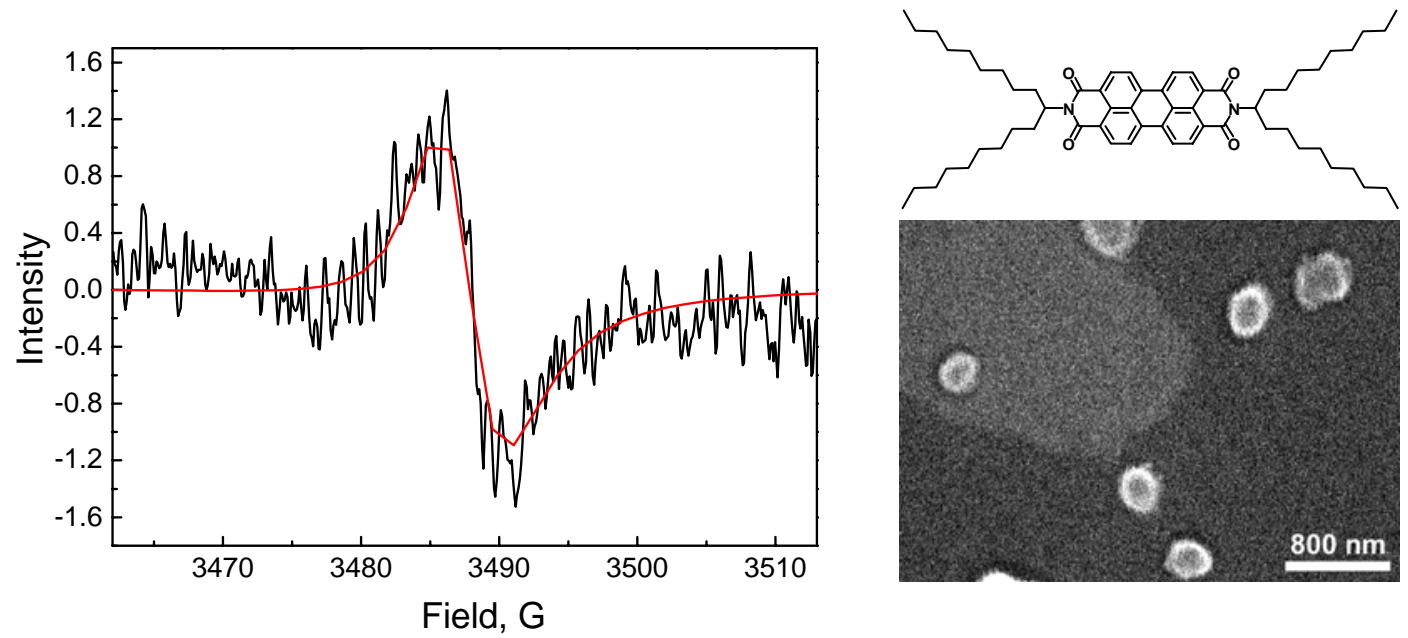

Figure S3. ESR spectrum of ND-PTCDI nanoparticles (adsorbed on cotton) immersed in hydrazine vapor. The weak signal is due to the limited amount of particles adsorbed on the cotton.

\section{Electrode fabrication and I-V measurement}

The electrical conductivity characterization of the nanobelt was based on direct currentvoltage (I-V) measurement, which was performed with a two-electrode device (with a single nanobelt deposited atop). The electrodes were fabricated by sputter deposition of gold ( $8 \mathrm{~nm}$ ) onto glass substrate using a Denton DESK IV sputter coater, followed by scratching the gold film with a razor blade to create a pair of film-electrodes with gap ranging from 50 to $100 \mu \mathrm{m}$ (Figure S4). The Pyrex glass substrate (from Corning Inc.) was pre-cleaned with piranha reagent (30:70 $\mathrm{H}_{2} \mathrm{O}_{2}(35 \%): \mathrm{H}_{2} \mathrm{SO}_{4}$ ), followed by rinsing with water and methanol. ${ }^{5}$ Caution: Piranha solution is an extremely strong oxidizing reagent. The roughness of the glass surface thus cleaned was about $0.8 \mathrm{~nm}$ as confirmed by AFM imaging. Such a flat surface is highly suitable for fabrication of flat electrodes and deposition of nanobelts.

To minimize the background current, the gap between the two electrodes was fabricated as large as possible. In this study most of the electrodes were fabricated with a gap around $80 \mu \mathrm{m}$, to still maintain feasible deposition of a single nanobelt onto the electrodes (the longest nanobelts synthesized can be as long as $\sim 150 \mu \mathrm{m}$ ). Prior to deposition of nanobelts, the fabricated electrodes were coated with a monolayer of $\alpha$-toluenethiol following the standard protocol developed previously. ${ }^{6}$ The monolayer thus coated enhances the hydrophobic interaction between PTCDI nanobelt and the electrode, and thus improves the contact between the two. Moreover, attachment of an oriented dipole layer to the electrode would decrease the Schottky energy barrier at the gold electrode, as previously observed for polymer/electrode interface. 
Herein, the Schottky energy barrier is mainly determined by the difference between the LUMO of PTCDI $(\sim-4.0 \mathrm{~V})$ and Fermi level of the gold electrode $(-5.3 \mathrm{~V})$. Enhancing the current through tuning the Schottky barrier was also achieved with single-molecule junctions of PTCDI molecules. ${ }^{8}$

Figure S5 (left) shows the optical microscope image of one typical device with a single nanobelt deposited across the electrode gap. The I-V measurement was carried out with a Keithley 6487 Picoammeter equipped with a Voltage Source providing applied bias between 0 $500 \mathrm{~V}$. The single-nanobelt device was placed in a sealed glass jar. After measuring the I-V curve for the pristine nanobelt, a small vial of hydrazine liquid was put into the jar, followed by $\mathrm{I}-\mathrm{V}$ measurement at different time intervals. After ca. $5 \mathrm{~min}$, the hydrazine vapor became saturated, and meanwhile the surface adsorption on nanobelt also reached the equilibrium as indicated by the saturation in current value. The maximal current thus obtained was plotted in Figure 3 to demonstrate the large current modulation of the nanobelt upon surface adsorption (doping), potentially leading to a new type of VOC sensing at nanoscale.

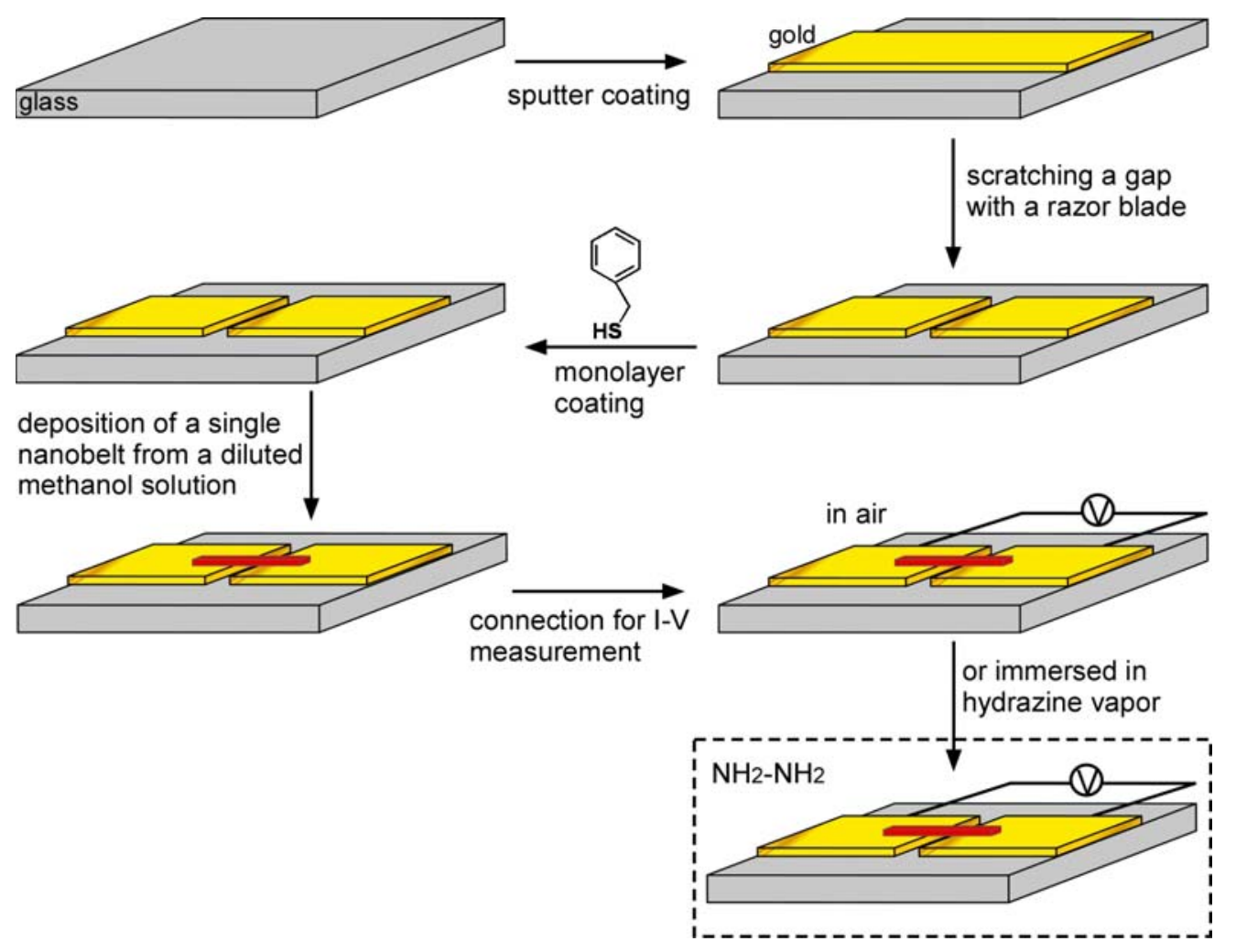

Figure S4. Step-by-step fabrication of a two-electrode device for characterizing the I-V (conductivity) property of PTCDI nanobelts. Enhancement of conductivity can be realized by surface electron doping by immersing the device in a saturated hydrazine vapor.

Interestingly, after repeated measurements (particularly at higher current, $>10 \mathrm{nA}$, in the presence of hydrazine) some the nanobelts were broken (as shown in Figure S5, right), in a manner similar to the breaking of a fuse wire. This observation is supportive to the existence of high current density, which was attained by external doping from hydrazine in combination with the effective $1 \mathrm{D}$ electronic delocalization along the nanobelt. 
The resistance of the PTCDI nanobelt was estimated from the linear region (at bias below 50 $\mathrm{V}$ ) of the I-V curve. With the known dimensional sizes of the nanobelts (width ca. $500 \mathrm{~nm}$, thickness ca. $100 \mathrm{~nm}$, and electrode gap $80 \mu \mathrm{m}$ ), the conductivity (or resistivity) of the nanobelt can be deduced following the standard definition.
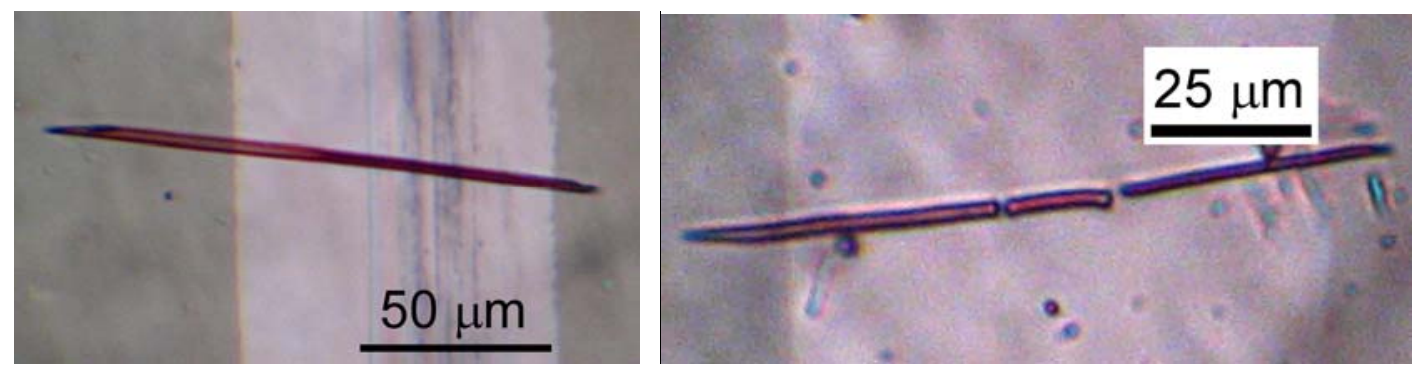

Figure S5. Optical microscope images of a single nanobelt freshly deposited atop the electrodes (left) and a broken single nanobelt after repeated test at high current under saturated hydrazine vapor (right).

(1) Langhals, H. Heterocycles 1995, 40, 477-500.

(2) Balakrishnan, K.; Datar, A.; Oitker, R.; Chen, H.; Zuo, J.; Zang, L. J. Am. Chem. Soc. 2005, 127, 10496-10497.

(3) Balakrishnan, K.; Datar, A.; Naddo, T.; Huang, J.; Oitker, R.; Yen, M.; Zhao, J.; Zang, L. J. Am. Chem. Soc. 2006, 128, 7390-7398.

(4) Chen, S.-G.; Branz, H. M.; Eaton, S. S.; Taylor, P. C.; Cormier, R. A.; Gregg, B. A. J. Phys. Chem. B 2004, 108, 17329-17336.

(5) Zang, L.; Liu, R.; Holman, M. W.; Nguyen, K. T.; Adams, D. M. J. Am. Chem. Soc. 2002, 124, 10640-10641.

(6) Sabatani, E.; Cohen-Boulakia, J.; Bruening, M.; Rubinstein, I. Langmuir 1993, 9, 2974-2981.

(7) Campbell, I. H.; Rubin, S.; Zawodzinski, T. A.; Kress, J. D.; Martin, R. L.; Smith, D. L.;

Barashkov, N. N.; Ferrariis, J. P. Physical Review B: Condensed Matter 1996, 54, R14321R14324.

(8) Xu, B. Q.; Xiao, X.; Yang, X.; Zang, L.; Tao, N. J. J. Am. Chem. Soc. 2005, 127, 2386-2387. 